\title{
AVALIAÇÃO DA FLEXIBILIDADE DOS ISQUIOTIBIAIS EM JOVENS SEDENTÁRIOS E IRREGULARMENTE ATIVOS DO ENSINO MÉDIO DE BELO HORIZONTE/MG.
}

\section{EVALUATION OF THE FLEXIBILITY OF ISCHIOTIBIALS IN YOUNG SEDENTARY AND IRREGULARY ASSESTS OF THE HIGH SCHOOL IN BELO HORIZONTE/MG.}

\author{
Renata Ribeiro da Silva1; Luciana Sucasas Vani Carneiro², Filipe Tadeu Sant Anna \\ Athayde $^{3}$, Carlos Alexandre Batista Metzker ${ }^{4}$
}

\section{RESUMO}

Os jovens do ensino médio têm sido caracterizados como grupo de risco para diversos tipos de afecções musculoesqueléticas em razão dos hábitos de vida adotados, especialmente pela permanência em posturas estáticas durante as longas jornadas de estudos. Foi objetivo desse estudo verificar a flexibilidade dos isquiotibiais (IT) de estudantes sedentários e irregularmente ativos de uma escola pública do ensino médio de Belo Horizonte/MG. Foi realizado um estudo observacional, transversal, analítico e quantitativo, que avaliou 27 estudantes do ensino médio, de ambos os sexos, com faixa etária entre 16 e 18 anos, classificados como sedentários e irregularmente ativos. A flexibilidade dos músculos IT, do lado dominante, foi mensurada por goniometria. Além de estatística descritiva, foi realizada uma análise comparativa da flexibilidade entre os sexos e de correlação linear considerando-se a idade. Todos os estudantes participantes do estudo (100\%) apresentaram algum grau de déficit de flexibilidade dos IT, sendo que a maioria $(59,3 \%)$ apresentou ADM de extensão do joelho avaliado variando entre 140 a 165 graus. Não houve correlação entre flexibilidade e idade e tampouco diferença significativa na comparação de flexibilidade entre os sexos. Ao final do estudo concluiu-se que todos os alunos participantes do estudo apresentaram déficit de flexibilidade dos IT e não houve significância estatística ao se considerar esses dados em relação ao sexo ou à idade.

Palavras-Chaves: Músculos Isquiossurais; Goniometria; Músculos, flexibilidade; Fisioterapia.

\footnotetext{
${ }^{1}$ Acadêmica do curso de Fisioterapia da Faculdade de Ensino de Minas Gerais - FACEMG, Belo Horizonte/MG, Brasil.

${ }^{2}$ Fisioterapeuta, Mestrado em Educação em Diabetes (Santa Casa de BH) e docente do curso de Fisioterapia da Faculdade de Ensino de Minas Gerais - FACEMG, Belo Horizonte/MG, Brasil.

3 Fisioterapeuta, Doutor em Ciências da Saúde (UFMG) e docente do curso de Fisioterapia da Faculdade de Ensino de Minas Gerais - FACEMG, Belo Horizonte/MG, Brasil.

${ }^{4}$ Fisioterapeuta, Mestrado em Administração (Fundação Pedro Leopoldo), coordenador e docente do curso de Fisioterapia da Faculdade de Ensino de Minas Gerais - FACEMG, Belo Horizonte/MG, Brasil.
} 


\section{ABSTRACT:}

The high school's youth has been characterized by group risk of hollow-muscles in reason of living habits, especially for keeping bad posture/ statics posture for long time of studying. The objective of this study was verify the flexibility of hamstrings of sedentary and unevenly active students on public school at Belo Horizonte/MG. An analytical and quantitative cross-sectional study was evaluated 27 high school students at the both gender and with 16-18 years old classified as sedentary and unevenly active. The hamstrings flexibility, of the dominant side, was measured using goniometria. In addition to descriptive statistics, a comparative analysis of flexibility between genders and a linear correlation considering age were performed. All high's school students who were analysed show degree of defict of hamstrings, although $59,3 \%$ has range orf motion of knee extention between 140-165 degrees. There was no correlation between flexibility and age, nor was there any significant difference in the comparison of flexibility between genders. At the end of the study, it was concluded that all students participating in the study had a deficit in hamstrings flexibility and there was no statistical significance when considering these data in relation to gender or age.

Keywords: Hamstring Muscles, Goniometry, Muscles, Flexibility, Physical Therapy Specialty

\section{INTRODUÇÃO}

O ensino médio caracteriza-se por uma das etapas finais da educação básica, onde os jovens buscam se inserirem na graduação. Nesta etapa os jovens passam mais tempo estudando e, com o avanço de tecnologias têm sido considerados grupos de risco para desenvolvimento de diversas alterações posturais em razão dos hábitos que se submetem e estilos de vida menos ativos ${ }^{1}$. A permanência em posturas estáticas e assentadas em frente a computadores e smartphones são alguns dos fatores que desencadeiam tais alterações e podem impactar sobre a flexibilidade, especialmente dos grupos da cadeia posterior².

Para que o individuo esteja apto fisicamente para realizar as tarefas do dia-adia se faz necessário um conjunto de fatores que possibilite mobilidade, qualidade de movimento e diminuição do risco de lesões, sendo a flexibilidade um componente muito relevante nesse contexto ${ }^{1}$. Caracterizada pela capacidade que o músculo tem de se alongar, permitindo que a articulação movimente-se por meio de uma amplitude de movimento (ADM) total ${ }^{3}$, a flexibilidade tem seu maior grau no ser humano ao nascimento, sendo gradualmente modificada progressivamente pelo envelhecimento ${ }^{4}$. 
Déficits na flexibilidade estão relacionados a problemas posturais, aumento da tensão articular, entre outros, sendo mais relevantes nos jovens o encurtamento muscular de isquiotibiais $(\mathrm{IT})^{5}$. Esse é um grupo de músculos biarticulados, composto pelo semitendinoso, semimebranoso e bíceps femoral, que estão envolvidos diretamente nos movimentos do quadril e joelho, tendo origem no osso isquiático e inserção nos côndilos medial e lateral do joelho ${ }^{6}$.

Por serem extensores primários do quadril, os IT desempenham fortes contrações para estabilizarem a pelve, durante a extensão do tronco e para controlarem a pelve sobre o fêmur à medida que o individuo em pé ou sentado, inclina-se para frente. Para tocar os pés e retornar a posição bípede, juntamente com a musculatura posterior do tronco constituem a cadeia muscular responsável pela manutenção e alinhamento posturais, na qual é de total relevância que a ação destes músculos estejam íntegras e sincronizadas ${ }^{7,8,9}$.

O encurtamento dos IT leva a uma diminuição do comprimento das suas fibras musculares e/ou tendíneas, influenciando em alterações posturais como inclinação da pelve e até mesmo distúrbios da marcha. Esse processo pode desencadear quadros álgicos nos membros inferiores e desalinhamento postural ${ }^{10,11}$. Assim, essas alterações causam comprometimento da biomecânica do movimento humano, o que também pode afetar secundariamente a funcionalidade, principalmente em atividades que envolvem as articulações da coluna lombar, quadris e joelhos ${ }^{12}$.

Considerando que os jovens estudantes do ensino médio são um grupo de indivíduos expostos a possíveis graus de encurtamento muscular dos IT, dado ao longo período que passam na posição sentada durante os estudos. As repercussões negativas que podem ocorrer do ponto de vista biomecânico e funcional nos mesmos, este estudo torna-se relevante na medida em que poderá servir como uma descrição da atual realidade desses indivíduos. Além disso, as alterações estruturais ou funcionais do corpo podem dar origem a novos desvios posturais, assim como desconforto, incapacidade e dor recorrentes ${ }^{13}$.

Diante do exposto, foi propósito deste estudo verificar a flexibilidade dos IT de estudantes sedentários e irregularmente ativos do ensino médio de uma escola estadual de Belo Horizonte/MG. 


\section{MÉTODOS}

Foi realizado um estudo observacional, transversal, analítico e quantitativo. A amostra do estudo foi obtida por conveniência a partir de alunos de uma escola pública estadual da região noroeste de Belo Horizonte MG, de ambos os sexos, que estudam quatro ou mais horas por dia na posição sentada e seja caracterizado como sedentário ou irregularmente ativo pelo International Physical Activity Questionnaire (IPAC).

Seguindo as normas preconizadas para as pesquisas envolvendo seres humanos do Conselho Nacional de Saúde (Resolução № 466, de 12/12/2012), o projeto de pesquisa foi aprovado pelo comitê de ética em pesquisa em seres humanos da Universidade Paulista - UNIP sob número 3.009.618.

Participaram do estudo 43 alunos, de ambos os sexos, com idade entre $16 \mathrm{e}$ 18 anos, que estudam quatro ou mais horas por dia na posição sentada e frequentavam, regularmente, a escola. Os voluntários leram e assinaram o termo de consentimento livre e esclarecido (TCLE) que as informavam sobre os procedimentos do estudo e riscos. Após a assinatura do TCLE os estudantes foram solicitados a responder um questionário, elaborado pelos pesquisadores, contendo questões sócio demográficas e que traçava o perfil físico dos mesmos. Em seguida, os participantes foram avaliados individualmente e classificados como sedentários e/ou irregularmente ativos pelo International Physical Activity Questionnaire (IPAC). Finalmente, os selecionados foram alocados para mensuração do grau de flexibilidade dos IT.

Indivíduos que por alteração estrutural apresentaram limitações de movimento articular nos joelhos e quadris ou articulações adjacentes, indivíduos com histórico de fratura não consolidada nos membros inferiores (MMII) ou com processo inflamatório agudo na articulação do joelho e/ou articulações adjacentes foram excluídos do estudo. Além disso, também não participaram àqueles que apresentaram sintomas que indicavam a presença de dores constantes, proximalmente, na articulação do joelho.

Antes do procedimento ser realizado, os alunos receberam explicações referentes à pesquisa. A avaliação foi realizada em um auditório disponibilizado pela diretoria da escola, sem expor o voluntário à perturbações externas. Para a realização dos procedimentos de avaliação da flexibilidade foi utilizado um 
goniômetro de acrílico manual da marca Arktus de $35 \mathrm{~cm}$, com sistema de transferidor de $0^{\circ}$ a $360^{\circ}$, possuindo uma régua com duas hastes para mensuração de amplitude articular dos músculos IT.

A mensuração da flexibilidade dos IT foi realizada com o estudante em uma maca, sendo posicionado em decúbito dorsal, com o quadril e joelhos estabilizados e fixados com o uso de duas faixas. O quadril e o joelho do lado predominante foram posicionados em flexão a 90 graus e o tornozelo na posição neutra. $O$ goniômetro foi posicionado considerando os pontos antropométricos do quadril, joelho e tornozelo, sendo adotado como ponto de referência (eixo) o côndilo lateral do fêmur, com braço fixo paralelo à superfície lateral e dirigida para o trocanter maior; e o braço móvel sendo paralelo à face lateral da fíbula e dirigido ao maléolo lateral ${ }^{14}$.

Seguidamente, foi solicitado a cada voluntário que realizasse extensão ativa do joelho, do lado dominante, até o ponto em que começasse a sentir um desconforto nos músculos IT. Foi considerada a flexão do joelho a $90^{\circ}$ como posição inicial e a extensão completa do joelho a 180 como posição final. Com base nos valores de referência para classificação de flexibilidade de extensão de joelhos foi considerado $180^{\circ}$ o parâmetro de normalidade ${ }^{15}$.

Após avaliação dos graus de flexibilidade dos IT, dos indivíduos participantes do estudo, os dados foram descritos por média e desvio padrão. Adicionalmente, foi realizada uma análise comparativa de flexibilidade entre os sexos por meio do teste T de student, uma vez que foi identificada normalidade na distribuição dos dados de flexibilidade; e de correlação linear entre flexibilidade e idade por meio do teste nãoparamétrico de Spearman devido à distribuição não-normal dos dados da variável idade. Foi considerado significativo $p<0,05$.

Uma semana anteriormente ao início da coleta de dados foi realizado um estudo piloto com a participação de sete alunos (cerca de $20 \%$ dos participantes do estudo), com o teste de confiabilidade, por meio do coeficiente de correlação de interclasse $(\mathrm{CCl})$, processado. A confiabilidade da medida intra-examinador foi classificada como excelente com o $\mathrm{CCl}$ de 0,97.

\section{RESULTADOS}

Do total de 84 alunos regularmente matriculados na escola, 43 aceitaram participar da pesquisa. Após análise dos critérios de exclusão a amostra reduziu-se 
a 27 alunos que concluíram a pesquisa (Figura 1). A amostra teve a maioria do sexo Feminino (66,6\%), da raça Pardo (44,4\%), e lado dominante sendo o direito (96\%).

Figura 1. Seleção de Amostra

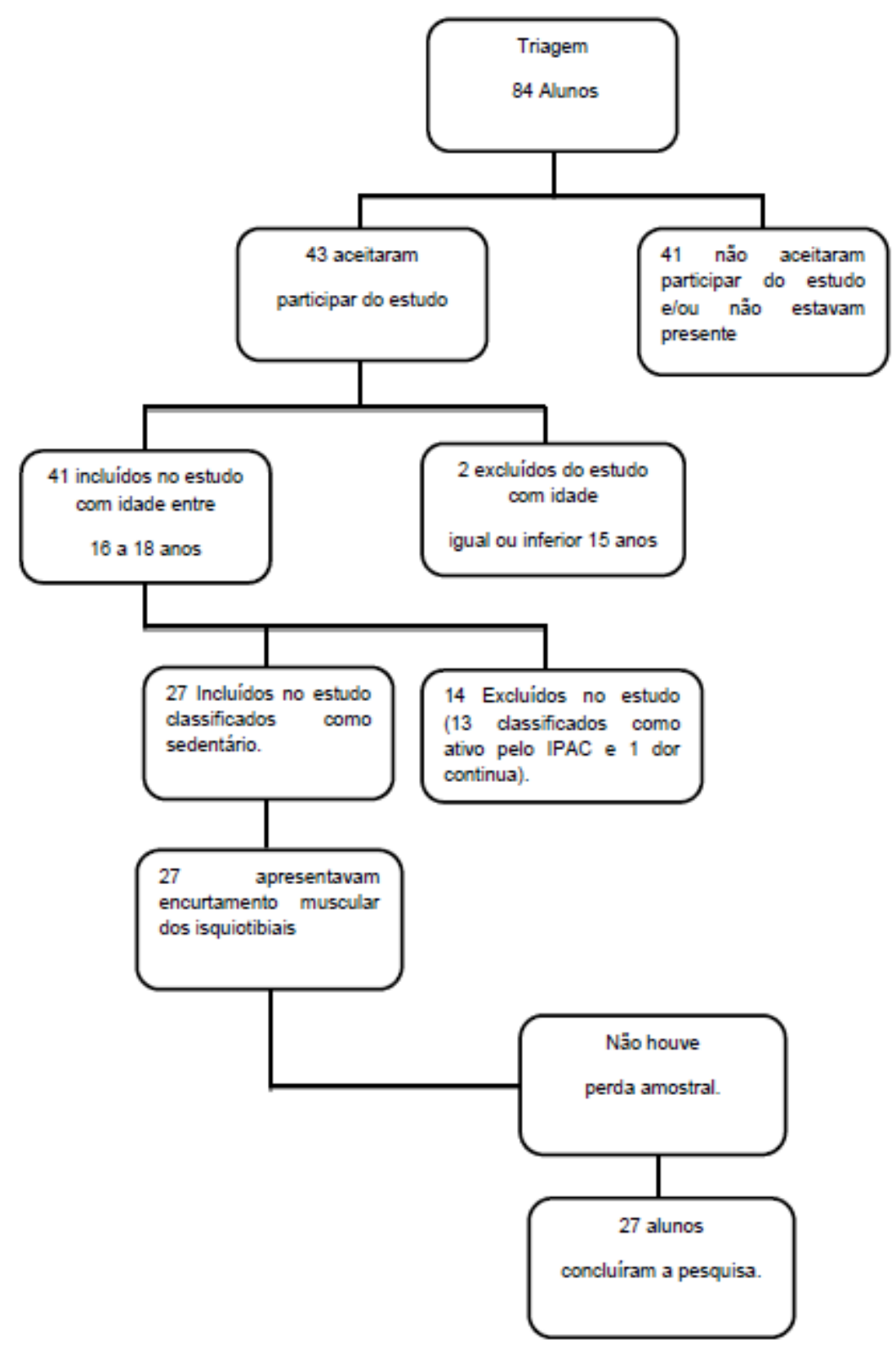

Fonte: Dados da pesquisa (2019).

Todos os participantes do estudo (100\%) apresentaram algum grau de limitação de flexibilidade dos IT, sendo que a maioria $(59,3 \%)$ apresentou ADM de extensão do joelho avaliado, variando entre 140 a 165 graus. Os resultados com o 
quantitativo de alunos e os respectivos graus de ADM avaliada podem ser observados na figura 2.

Figura 2. Graus de Flexibilidade dos IT da amostra estudada

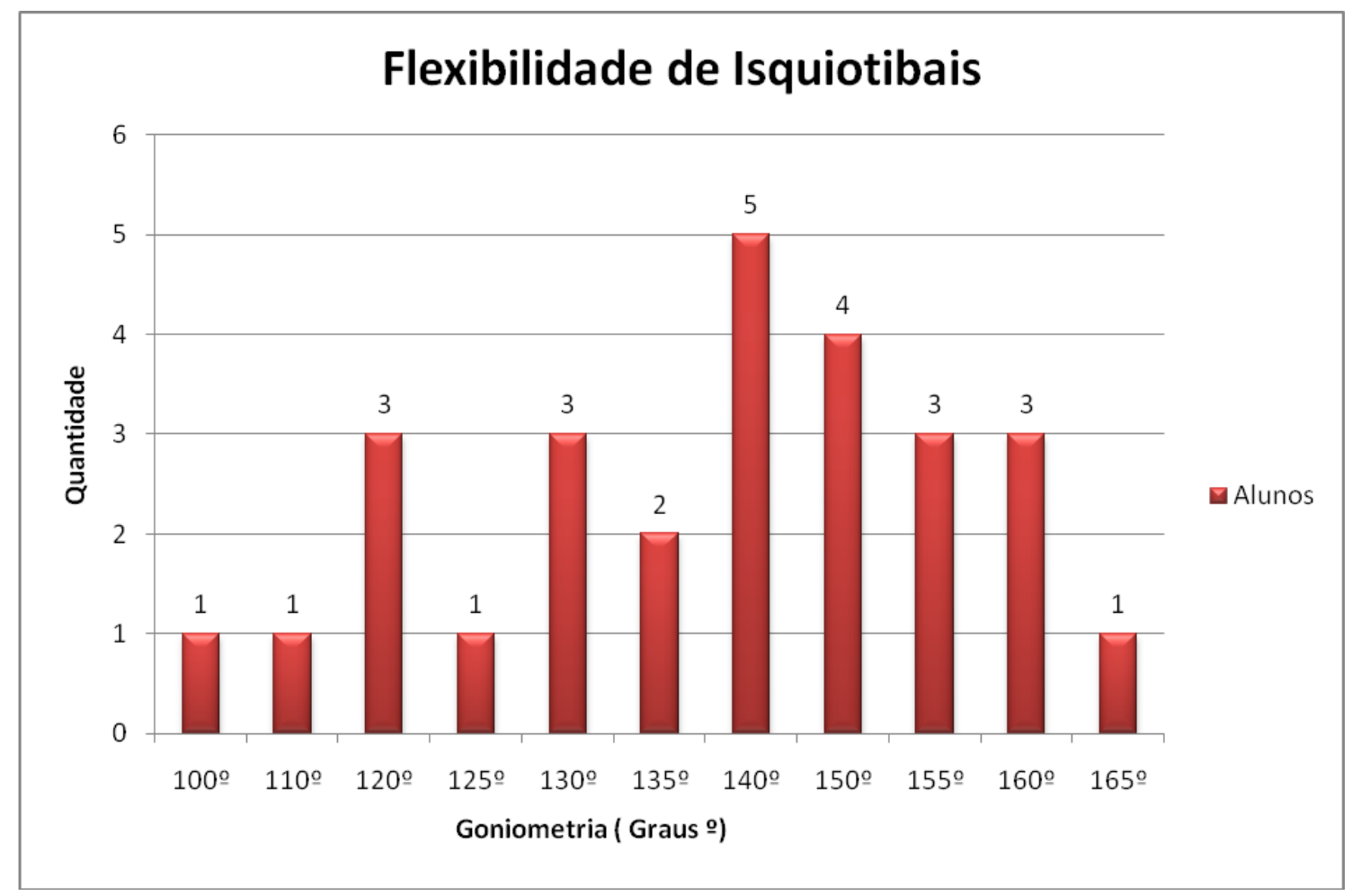

Fonte: Dados da pesquisa (2019).

Não houve correlação entre déficit de flexibilidade dos IT e idade e na comparação entre déficit de flexibilidade e sexo não houve diferença estatisticamente significante (Tabela 1).

Tabela 1 - Análise da flexibilidade de IT comparada com sexo e correlacionada com idade dos participantes do estudo.

\begin{tabular}{ccc}
\hline & Sexo & Idade \\
\cline { 2 - 3 } Flexibilidade dos IT & $p=0,73$ & $p=0,33$ \\
& $r=0,21$ \\
\hline
\end{tabular}

Nível de significância $\mathrm{p}<0,05$.

Fonte: Dados da pesquisa (2019). 


\section{DISCUSSÃO}

Dos 27 alunos do ensino médio avaliados no estudo, classificados como sedentários e/ou irregularmente ativos pelo IPAC, 100\% apresentaram encurtamento de IT, sendo 15 do sexo feminino e 12 do sexo masculino. Este resultado aponta para um grande índice de encurtamento muscular na amostra estudada, situação também constatada nos estudos de Guadagnine et. al/3 Pinto et. al2; e Santos et. $a l^{15}$ que também analisaram o encurtamento de IT em adultos jovens.

No presente estudo, independentemente do sexo dos participantes, todos apresentaram algum grau de encurtamento de IT. No entanto, os trabalhos de Pinto et. $a l^{2}$; e Santos et. a $/^{15}$ indicaram haver maior flexibilidade em mulheres do que os homens. Para os autores as variações hormonais alteram a fisiologia feminina e pela ação estrógena, normalmente, indivíduos do sexo feminino apresentam melhores níveis de flexibilidade. $O$ achado diferente no presente estudo pode estar relacionado ao tamanho da amostra e por ser composta integralmente por adultos jovens de ambos os sexos.

Fatores endógenos como a idade e a individualidade biológica influenciam na flexibilidade. Esta, concomitantemente, manifesta-se de forma diferente em crianças, adolescentes e adultos, tendendo a diminuir com a idade ${ }^{16,17}$. No entanto, os resultados obtidos no presente estudo não confirmam essa teoria, na medida em que não houve correlação entre a idade dos participantes e flexibilidade dos IT. No presente estudo $100 \%$ dos participantes apresentaram algum grau de limitação de flexibilidade, sendo que $46,51 \%$ tiveram resultado de ADM de extensão do joelho avaliado somente até 140 graus.

No presente estudo foi realizada a avaliação da flexibilidade com utilização da goniometria de forma ativa. Contudo, sabe-se que outros instrumentos também podem ser utilizados para mensuração do grau de flexibilidade. O estudo de Carregaro et. a/5 realizou as mensurações com utilização da fotogrametria associada ao teste do terceiro dedo ao chão e de elevação dos MMII. Já Chagas e Bhering ${ }^{18}$ sugerem que a mensuração da flexibilidade envolvendo os músculos posteriores da coxa podem ser feita por um equipamento denominado banco para a avaliação da flexibilidade (Welss). Tanto o teste do terceiro dedo ao chão quanto a utilização do banco de wells são formas de avaliação passiva da flexibilidade dos IT. Cabe ressaltar que a forma passiva de avaliação da flexibilidade dos IT não envolve 
contração muscular dos músculos da coxa. Já a forma ativa, usada neste estudo, envolve a força muscular dos quadríceps para estender o joelho. A diminuição de força muscular desse grupo muscular pode levar a uma diminuição do grau de extensão do joelho, assim como uma consequente interferência na flexibilidade plena dos IT. Sendo assim, as formas passivas são comumente mais indicadas para avaliar alteração de flexibilidade muscular.

No presente estudo, do total dos alunos 43 alunos que aceitaram participar da pesquisa, $62,79 \%$ (27) compuseram a amostra por se enquadrarem nos grupos sedentários ou irregularmente ativos segundo o IPAC. Sabe-se que a falta de atividade física correlacionada com posturas inadequadas pode ser um fator causal para o encurtamento de IT. Diferentemente desse estudo, o trabalho de Pinto et. $\mathrm{al}^{2}$, apresentou que a maioria dos escolares praticava atividade física fora das aulas de Educação Física, com frequência de uma a três vezes por semana com mais de uma hora de duração. No mesmo estudo pode-se observar que apenas $36,5 \%$ dos que praticam atividade física regular estavam na faixa de condição de risco à saúde na flexibilidade do quadril. Quando a a análise inferia os alunos que não praticavam, esse número subiu para $45,8 \%$.

Já Melo et.al19 descrevem a possibilidade de não haver relação importante entre o nível de atividade física e a flexibilidade entre os jovens. Os autores ainda apontam que não o nível de atividade física, mas sim o padrão da mesma possa intervir na flexibilidade. Consideram a possibilidade de que determinados movimentos específicos possam produzir aumentos ou mesmo a manutenção de uma elevada mobilidade em uma determinada articulação, mas não no corpo como um todo.

Por estar relacionada a uma etapa em que os alunos do ensino médio adotam posturas inadequadas e viciosas, permanecendo também longos períodos com atividades extra-classe, a condição temporal pode explicar o fato de todos, selecionados no presente estudo, terem apresentado algum grau encurtamento de IT. Situação que corrobora com os achados de Santos et. al ${ }^{15}$.

\section{CONCLUSÃO}

Ao final do estudo concluiu-se que todos os alunos participantes do estudo apresentaram déficit de flexibilidade dos IT, ao considerar como referencial de 
encurtamento as amplitudes de movimento inferiores a $180^{\circ}$. O presente estudo também não identificou, quanto à flexibilidade dessa musculatura, significância na correlação com idade ou comparação entre sexos.

\section{REFERÊNCIAS}

1. Cezar JR, Cezar MR. Relação entre níveis de flexibilidade e composição corporal de jovens praticantes de futebol. Unoesc \& Ciência - ACBS - Edição Especial 2014; 121-8.

2. Pinto MG, Soares A, Machado Z, Azevedo SF, Kraeski AC, Folle A, Guimarães ACA. Nível de flexibilidade de alunos do ensino médio de Florianópolis -SC: uma análise centrada no sexo, idade e prática de atividade física extraescolar. Rev. Bras. Educ. Fís. Esporte 2013; 27(4):657-65.

3. Aquino CF, GonçalvesGGP, Fonseca ST, Mancini MC. Análise da relação entre flexibilidade e rigidez passiva dos isquiotibiais. Rev. Bras. de Medicina do Esporte. 2006; 12(4):195-200.

4. Vale RGS, Aragão JCB, Dantas EHM. A flexibilidade na autonomia funcional de idosas independentes. Fitness \&Performance Journal. 2003; 2(1): 29-3.

5. Carregaro RL, Silva LCCB, Gil Coury HJC. Comparação entre dois testes clínicos para avaliar a flexibilidade dos músculos posteriores da coxa. Revista Brasileira de Fisioterapia. 2007; 11(2): 139-45.

6. Almeida TT, Jabur MN. Mitos e verdades sobre flexibilidade: reflexões sobre o treinamento de flexibilidade na saúde dos seres humanos. 2007; 3(1): 337-44.

7. Cardoso JHP, Damasceno DT, Camara DT, Miranda FHS; Assis LC; Silva EAL; Klebis LO; MNoreno ACR.. Análise de encurtamento dos músculos isquiotibiais em adultos jovens de 18 a 25 anos. Colloquium Vitae, 2016; 8:p.90-6.

8. Veiga PH, Daher CR, Morais MF. Alterações posturais e flexibilidade da cadeia posterior nas lesões em atletas do futebol de campo. Rev.Bras.Cienc Esporte. $2011 ; 33: 235-48$.

9. Azevedo DG, Silva MR. Prevalência de encurtamento dos músculos isquiotibiais em universitários. Revista Inspirar: Movimento \& Saúde. 2010; 2(4): 95-103, 2010. 
10. Polachini LO, FusakiL, Tamoso M, Tellini GG, Masiero D. Estudo comparativo entre três métodos de avaliação do encurtamento de musculatura posterior de coxa. Revista Brasileira de Fisioterapia. 2005; 9(2): 187-93.

11. Coelho JJM, Graciosa MD, Medeiros DL, Pacheco SCS, Costa LMR, Ries LGK. Influência da flexibilidade e sexo na postura de escolares. Revista Paulista de Pediatria. 2014; 32(3):223-8.

12. Cintra VFMC, Nicolau AR, Júnior PRA, Uchôa MMS, Veiga AHP. Influência da laserterapia associada ao alongamento passivo sobre o músculo isquiotibial. Revista ConScientiae Saúde. 2012;11(3):498-505.

13. Guadagnine $P$, Olivoto R. Comparativo de Flexibilidade me idosos praticantes e não praticantes de Atividades Físicas. Revista Digital. Buenos Aires: n.69, fev. 2004. Disponível em [http://www.efdeportes.com: Acesso on line: 11 nov.2019].

14. Marques AP. Manual de Goniometria. 2. Ed. Editora Manole, 2003.

15. Santos S, Pires AHB, Vieira RCS. Avaliação da flexibilidade da cadeia posterior da coxa em universitárias. RSCM, 2018; 1:1-13.

16. Minatto G, Ribeiro, RR., Achour JA, Santos KD. Idade, maturação sexual, variáveis antropométricas e composição corporal: influências na flexibilidade. Rev Bras Cineantropom \& Desempenho Hum. 2010, 12(3):151-58.

17. Achour JA. Flexibilidade e alongamento: saúde e bem-estar. São Paulo: Manole; 2004.

18. Chagas $\mathrm{MH}$, Bhering EL. Nova proposta para avaliação da flexibilidade. Rev Bras Educ Fís Esporte 2004;18(3):239-48.

19. Melo FAP, Oliveira FMF, Almeida MB. Nível de atividade física não identifica o nível de flexibilidade de adolescentes. Rev Bras Ativ Fís Saude. 2009; 14(1):48-54. 